\title{
Axisymmetric vortex breakdown. Part 3 Onset of periodic flow and chaotic advection
}

\author{
By J. M. LOPEZ AND A. D. PERRY† \\ Aeronautical Research Laboratory, 506 Lorimer Street, Fishermens Bend, Victoria 3207, \\ Australia
}

(Received 26 March 1991 and in revised form 1 July 1991)

\begin{abstract}
When the fluid inside a completely filled cylinder is set in motion by the rotation of one endwall, steady and unsteady axisymmetric vortex breakdown is possible. Nonlinear dynamical systems theory is used to describe the changing kinematics of the flow as the speed of the rotating endwall is increased. Two distinct modes of oscillation have been found in the unsteady regime and the chaotic advection caused by the oscillations has been investigated. The results of this study are used to describe the filling and emptying processes of the vortex breakdown bubbles observed in flow visualization experiments.
\end{abstract}

\section{Introduction}

Complex fluid motions may result when the fluid inside a completely filled cylinder is driven by the constant rotation of the bottom endwall. At low rotation rates the flow is steady and axisymmetric vortex breakdown is possible. This regime has been explored experimentally (Vogel 1968; Escudier 1984), as well as numerically (Lugt \& Abboud 1987; Lopez 1988, 1990) and the dynamics of the steady flow is understood (Brown \& Lopez 1990). Vortex breakdown in the cylinder flow is a result of the production of an azimuthal component of vorticity which induces a reversed flow on the axis and hence, by continuity, a local divergence of the streamtubes in the neighbourhood of the reversed axial flow. This is produced by the tilting and stretching of the vorticity vector which consists primarily of an axial component due to the swirling motion and an azimuthal component due to the meridional circulation. In general, the velocity vector will not be aligned with or perpendicular to the vorticity vector. If the ratio of the helix angle of the velocity vector to that of the vorticity vector is greater than one (the helix angle of a vector is the ratio of its azimuthal and axial components), then, as shown in Brown \& Lopez (1990), the tilting and stretching of vorticity leads to the production of an azimuthal component of vorticity inducing a reversed axial flow, i.e. the vortex breakdown of the central vortex.

In certain regions of parameter space, the flow inside the cylinder is unsteady. Two distinct modes of periodic oscillation have been identified. One consists of two regions enclosed by separatrices of the instantaneous streamline pattern, periodically coalescing and separating. The other consists of a wave travelling axially from top to bottom each period. This picture of the instantaneous streamlines is markedly different from flow visualizations of dyelines. Flow visualizations, consisting of

$\dagger$ Present address: Graduate Aeronautical Laboratories, California Institute of Technology, Pasadena, CA 91125, USA. 
dyelines (which approximate streaklines very well when the properties of the dye are not too different from those of the fluid), show the formation of long thin 'fingers' entering and leaving the 'bubble' regions.

The marked difference between the Eulerian viewpoint (from the instantaneous streamlines) and the Lagrangian viewpoint (from the streaklines) in unsteady flows is well known (Hama 1962; Stuart, Pankhurst \& Bryer 1963), and yet interpretation of flow visualizations of unsteady flows are often confused and occasionally incorrect. Neitzel (1988) has shown numerically how in the unsteady axisymmetric swirling flow inside a cylinder driven by a rotating endwall, a streakline released near the axis can give the visual impression of a non-axisymmetric flow.

Here the periodic flow is examined from both an Eulerian and Lagrangian point of view. The Lagrangian viewpoint is found to be particularly enlightening. The particle equations projected onto a meridional plane are shown to be Hamiltonian and use is made of Hamiltonian dynamical systems theory to describe in detail the complicated fluid motions, in particular the filling and emptying processes of the vortex breakdown 'bubbles'.

A typical sequence of bifurcations in the topology of the steady and unsteady flows is described. The only reported experimental results in the unsteady regime are those of Escudier (1984), but that report gives very little detail of the unsteady flow. A number of numerical investigations have included the unsteady regime (Lopez 1989; Daube \& Sorensen 1989), but the results from these investigations were presented in terms of an Eulerian description.

The use of dynamical systems theory to describe the fluid motions for swirling flows undergoing unsteady vortex breakdown is not new. Holmes (1984) outlined the potential of such an application. He envisaged a bifurcation of the steady axisymmetric flow resulting from non-axisymmetric, time-periodic perturbations leading to chaotic streaklines in the vortex breakdown flow which, he expected, would account for the complex fluid motions observed experimentally by Faler \& Leibovich (1977).

Rather than perturbing the steady flow by some external forcing, the NavierStokes equations are solved here for a swirling flow which is allowed to undergo a transition to a periodic state as the Reynolds number is increased. Next, a Poincaré mapping is defined for this flow and its topology (i.e. the Lagrangian representation of the flow) is analysed. Further, changes in the topology are related to changes in the Eulerian representation. This is an enlightening analysis which is not often performed, particularly in view of the fact that the transitions are not externally forced. Many of the ideas proposed by Holmes (1984) are found in this study of swirling flow undergoing vortex breakdown. The main difference between Holmes' scenario and that found in the cylinder flow is that the periodic flow is axisymmetric, as shown experimentally by Escudier (1984), whereas in Holmes' scenario, the bifurcation to periodic flow was associated with symmetry breaking as well. However, his prediction of the fluid motion in the 'bubble' of a vortex breakdown, with thin 'fingers' (lobes) originating upstream being 'entrained' into the interior of the 'bubble', where they circulate at least once, and similarly fluid originating inside the 'bubble' leaving the 'bubble', is the type of motion found in the cylinder flow. Most of the dynamical systems theory concepts and ideas used here can be found in Guckenheimer \& Holmes (1986) and the theory of the lobe dynamics is given in a recent article by Rom-Kedar \& Wiggins (1990). Lichtenberg \& Lieberman (1983), Wiggins $(1988,1990)$ and Ottino (1989) also provide good introductions to the subject. 


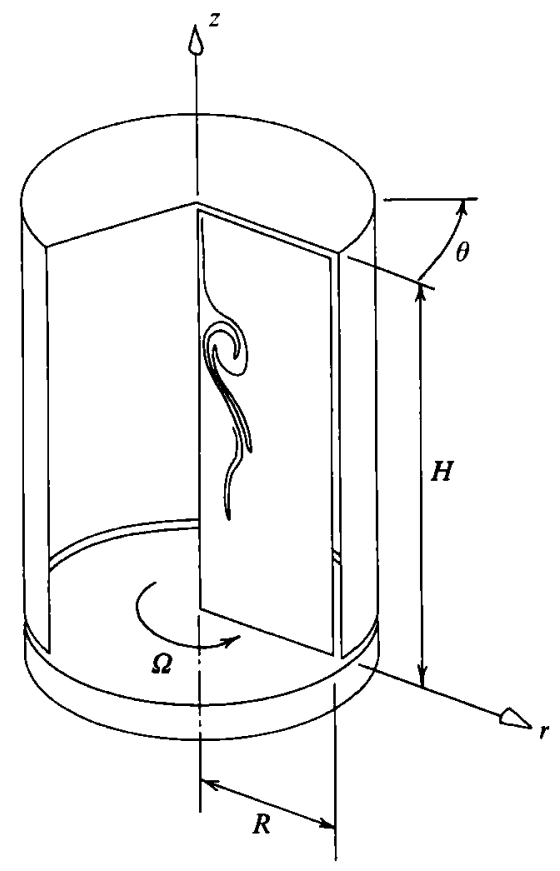

Figure 1. A schematic of the cylinder flow showing the intersection of a streaksheet with a meridional plane.

\section{Swirling flow in an enclosed cylinder}

Consider a circular cylinder, of radius $R$ and height $H$, completely filled with an incompressible fluid of constant kinematic viscosity, $\nu$. At time $t=0$, when the fluid and the cylinder are at rest, the bottom endwall is impulsively set to rotate at a constant angular speed $\Omega$. This flow, first investigated experimentally by Vogel (1968) and later by Ronnenberg (1977) and Escudier (1984), is completely specified by two non-dimensional parameters. These are the aspect ratio of the cylinder $H / R$, and the rotational Reynolds number $R e=\Omega R^{2} / \nu$.

When the bottom endwall is impulsively started, a thin Ekman boundary layer (with constant thickness of order $R e^{-\frac{1}{2}}$ ) is formed which centrifuges fluid outwards while drawing fluid in from above to maintain continuity. The expelled fluid then spirals up the sidewall, forming another boundary layer from which a portion of the angular momentum and total head acquired in the Ekman layer is transferred to the interior flow through the action of viscous stresses. This sidewall boundary layer is deflected at the upper endwall and an upper endwall boundary layer is formed. The endwall boundary layer separates at $r=0$, forming a central vortex which returns fluid back towards the Ekman boundary layer. The central vortex, whose structure is primarily determined by the structure of the stationary endwall boundary layer, is broad and diffuse for $R e<1000$. At these low Reynolds numbers, the central vortex is essentially cylindrical and its associated vorticity vector is primarily directed in the axial direction, with a relatively small azimuthal component due to the meridional circulation. In a region of $(R e, H / R)$ parameter space, this central vortex undergoes vortex breakdown, as detailed in Lopez (1990) and Brown \& Lopez (1990). A schematic of the flow is shown in figure 1.

The details of the computational technique used to calculate the flow field are given in Lopez (1990). The axisymmetric form of the Navier-Stokes equations is 
integrated using a time-accurate finite-difference technique. The restriction to axial symmetry is supported by Escudier's (1984) experiments where the periodic flow remained axisymmetric.

\section{A dynamical systems representation of the flow}

A simple Lagrangian representation of the flow can be obtained by exploiting its axial symmetry and temporal periodicity (at least in the region of parameter space being considered).

\subsection{Axisymmetric particle equations}

The particle equations for incompressible two-dimensional planar flow are Hamiltonian. The particle equations for incompressible axisymmetric flow can also be cast as a Hamiltonian system.

In an axisymmetric flow, an axially centred circular ring of fluid remains axially centred and circular as it evolves in time. The only effect of swirl (i.e. non-zero azimuthal component of velocity) is to rotate the ring about the axis of symmetry. Rather than following the motion of particles in three dimensions, it is sufficient to study the motion of ring intersection points in an arbitrary meridional plane $(r, z)$, where $(r, \theta, z)$ are the usual cylindrical coordinates. If $(u, v, w)$ is the corresponding velocity field, then

$$
\dot{r}=u=-\frac{1}{r} \frac{\partial \psi(r, z, t)}{\partial z}, \quad \dot{z}=w=\frac{1}{r} \frac{\partial \psi(r, z, t)}{\partial r}
$$

describes the motion of the intersection point, where $\psi$ is the Stokes stream function. By the coordinate transformation $p=\frac{1}{2} r^{2}$ (Benjamin 1962), the system (1) becomes

$$
\dot{p}=-\frac{\partial \psi}{\partial z}, \quad \dot{z}=\frac{\partial \psi}{\partial p},
$$

which constitutes a Hamiltonian system of one degree of freedom. If the flow is steady the system (2) is also autonomous. In the unsteady (non-autonomous) case, chaotic solutions to $(2)$ are possible. The velocity field $(\dot{p}, \dot{z})$ is divergence free and hence area preserving in the canonical $(p, z)$ phase space.

\subsection{The Poincaré map}

For a periodic flow

$$
\psi(r, z, t)=\psi(r, z, t+\tau)
$$

where $\tau$ is the period of the flow. Equation (3) is also true in the steady case, the value of $\tau$ being arbitrary. The periodicity is exploited by defining a Poincaré map:

$$
\left(r\left(t_{0}\right), z\left(t_{0}\right)\right) \mapsto\left(r\left(t_{0}+\tau\right), z\left(t_{0}+\tau\right)\right) .
$$

Put simply, the Poincaré map maps the position of ring intersection points in $(r, z)$ space at time $t_{0}$ to their location one period later. The image of a point $x$ under $n$ applications of the map is denoted by $F^{n}(x)$. The point mapped to $x$ under $n$ applications of the map is denoted $F^{-n}(x)$ and is well defined since the map is invertible. A point $\boldsymbol{x}$, such that $\boldsymbol{F}(\boldsymbol{x})=\boldsymbol{x}$, is a fixed point of the Poincaré map. All possible maps can be obtained by choosing $0 \leqslant t_{0}<\tau$ and the topology of the map is independent of the choice made.

A streaksheet is obtained when dye is continuously released from a circular ring centred on the axis of symmetry. The intersection of a streaksheet with an arbitrary meridional plane defines a line in $(r, z)$-space. These lines behave like streaklines in 
a planar two-dimensional flow and henceforth shall be called streaklines. These streaklines are revealed in flow visualization experiments, e.g. Escudier (1984), when dye is injected at $r=0, z=H$ and a meridional plane is illuminated by a laser sheet. A physical interpretation of the Poincaré map is sometimes given in terms of streakline patterns. To make the connection between these streaklines and the map precise the definition of these streaklines is extended. Usually, a streakline represents the positions of particles released from a particular point at some past time. Here, streaklines represent the positions of intersection points of rings rather than the positions of particles. Let these streaklines also include the intersection points of rings which will occupy the release position at some future time. This 'backwards' part of the streakline can be imagined as the line obtained when dye is released in a time-reversed version of the flow. Streaklines so defined are infinitely long in both directions (except in special cases, e.g. when they terminate or originate at fixed points) and when sampled at the phase of the Poincaré map, are invariant curves of the Poincaré map, i.e. they map to themselves. A point on a streakline is always mapped to another point on the same streakline. A streakline pattern is a 'phase portrait' of the Poincaré map.

If the Poincare map is defined for $(p, z)$-space rather than $(r, z)$-space it is also area preserving. This is particularly relevant when considering lobe dynamics (RomKedar \& Wiggins 1990; Rom-Kedar, Leonard \& Wiggins 1990). An element of area in $(p, z)$-space is $\delta p \delta z=r \delta r \delta z$ and the volume of a torus of cross-sectional area $\delta r \delta z$ in $(r, \theta, z)$-space is $2 \pi r \delta r \delta z$. Hence, while the Poincaré map in $(r, z)$-space is not area preserving; it is volume preserving when considered as a meridional cross-section of a toroidal volume.

\section{Résumé of the steady flow}

The steady flows described in Lopez (1990) for $H / R=2.5$ are briefly reviewed with emphasis placed on the development of the topology as $R e$ is increased. This leads naturally into the unsteady regime and an analysis of the kinematics of the periodic flow.

\subsection{Topology of the steady flow}

In steady flow streamlines, streaklines and particle paths coincide and are invariant curves of the Poincaré map. The fixed points of the Poincaré map can be classified according to their stability. For a two-dimensional area-preserving map the only possible fixed points are hyperbolic (unstable) and elliptic (Liapunov stable), apart from the degenerate parabolic fixed points on the rigid boundaries. A rule relating the number of saddles and centres in instantaneous streamline patterns can be easily derived using the Poincaré-Bendixson (or 'hairy sphere') theorem (Davey 1971; Hunt et al. 1978). For the meridional flow the number of saddles must equal the number of centres. A saddle on the $r=0$ axis or no-slip boundary counts as half a saddle and each corner counts as a quarter. An heuristic derivation of a similar rule for fixed points of the Poincaré map is presented in the Appendix.

Let the position of a particle which moves on a closed orbit in $(r, \theta, z)$-space be called a periodic point of the flow. Let the order of such a point be the number of orbits about the $r=0$ axis it takes the particle to return to its starting position. It is worthwhile noting when a fixed point of the Poincaré map is not a periodic point of the unsteady flow. All fixed points on the $r=0$ axis are obviously periodic points of the flow. However, a particle placed at a fixed point off the axis, located at $\left(r_{1}, \theta_{1}, z_{1}\right)$, will end up at location $\left(r_{1}, \theta_{2}, z_{1}\right)$ after one period of the periodic flow. If $\left(\theta_{2}-\theta_{1}\right) / \pi$ is rational, 


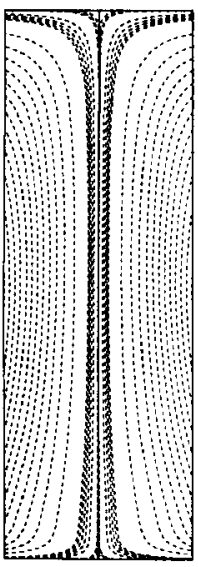

$\operatorname{Re}=1000$

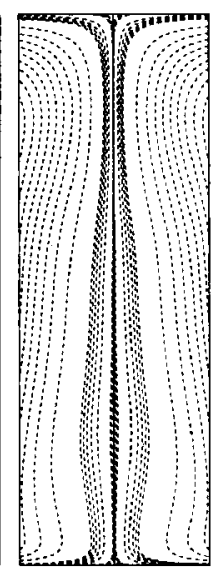

1600

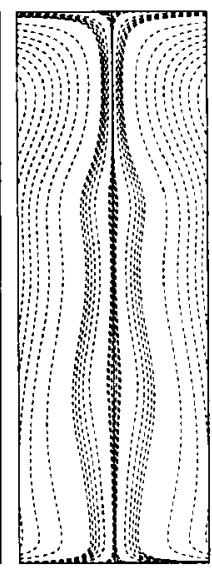

1800

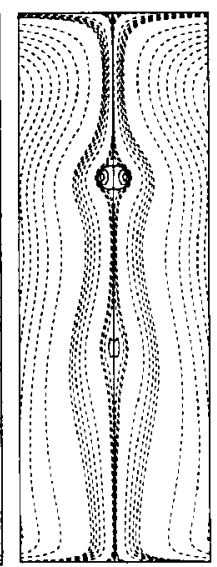

1918

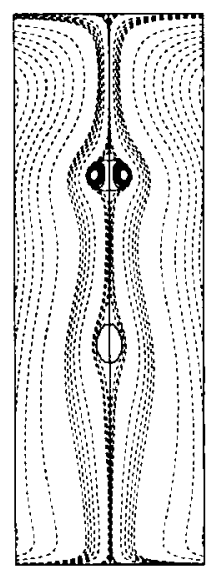

1942

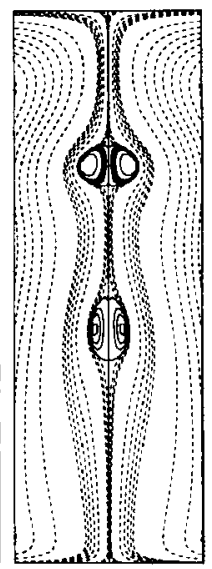

2000
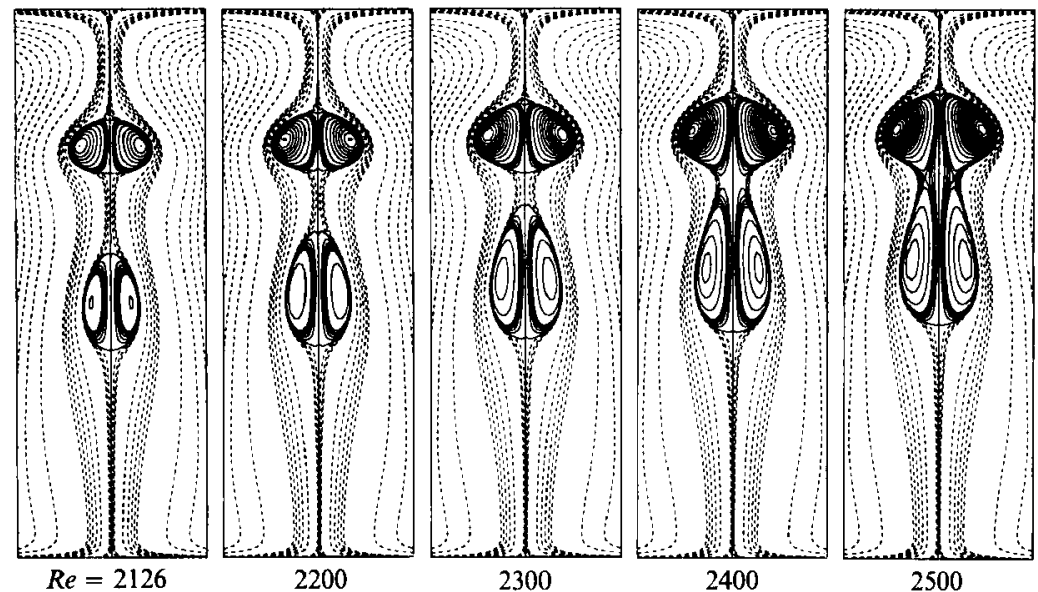

Figure 2. Details of the stream function at steady state $\left(0 \leqslant r \leqslant \frac{5}{12} R\right)$ for $H / R=2.5$ and $R e$ as indicated $\left(n_{r}=61, n_{z}=151\right.$ and $\left.\delta t=0.05\right)$.

then the fixed point of the map is also a periodic point of the flow (possibly of high order); but if $\left(\theta_{2}-\theta_{1}\right) / \pi$ is not rational, then the point is not a periodic point of the flow of any order.

For the steady swirling flow, the only fixed points of the map which correspond to particles which never move in $(r, \theta, z)$-space, are the stagnation points on the axis of symmetry and of course, the stationary boundaries. All other hyperbolic and elliptic fixed points occur off the axis where the azimuthal velocity is non-zero and hence are periodic points of the steady flow. The time taken to complete one orbit is determined by the local azimuthal velocity.

The development with increasing $R e$ of the steady flow's topology in the central region (i.e. $r \leqslant \frac{5}{12} R$ ) is shown in figure 2 for $H / R=2.5$. As mentioned above, for these steady flows the level curves of the stream function give a phase portrait of the Poincaré map, of which they are invariant curves. For $R e<1918$, the only fixed point of the map is elliptic, corresponding to the centre due to the meridional circulation (it is not shown in figure 2 as it lies at $r>\frac{5}{12} R$ ). Note that owing to the axial symmetry, the flow only needs to be considered in the half-plane $r \geqslant 0, \theta=\theta_{0}$, although the plane $\theta=\theta_{0}+\pi$ is included in the figures. For $R e \geqslant 1918$, the central 
(a)

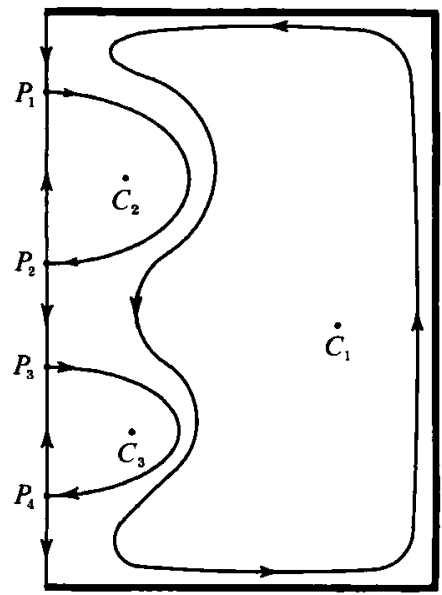

(b)

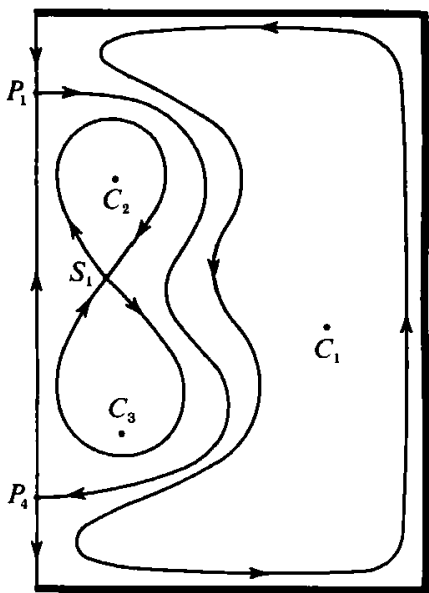

Figure 3. Schematics of two topologies occurring in the steady regime of the vortexbreakdown flow.

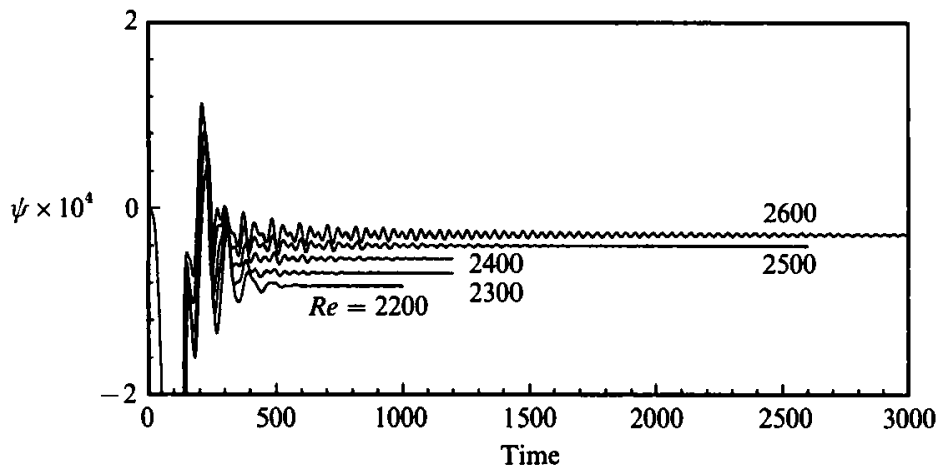

Figure 4. Time history of the stream function at the point $r=\frac{1}{6} R, z=\frac{2}{3} H$ for $H / R=2.5$ and $R e$ as indicated (one time unit is one rotation of the bottom endwall).

core develops a series of stagnation points resulting from its vortex breakdown. Details of this process and the physical mechanisms involved are given in Lopez (1990) and Brown \& Lopez (1990).

For $1918 \leqslant R e<2300$, the topology of the Poincaré map consists of four hyperbolic fixed points (stagnation points) on the axis and three elliptic fixed points (centres) off the axis. Figure $3(a)$ is a schematic of this. There are four special invariant curves originating or terminating at each hyperbolic fixed point. In the terminology of dynamical systems, these are the stable and unstable manifolds. Points on the two stable manifolds, $W^{s}$, asymptote towards the fixed point under repeated applications of the map. Points on the two unstable manifolds, $W^{u}$, asymptote towards the fixed point under repeated applications of the inverse map. In steady flow, a separatrix connecting the two hyperbolic fixed points on the axis, corresponding to the two stagnation points of the flow, is an unstable manifold for one and a stable manifold for the other. When the separatrix connects two different hyperbolic fixed points, the orbit is heteroclinic and when the separatrix connects a hyperbolic fixed point to itself the orbit is homoclinic. Stable manifolds cannot intersect themselves or each other. The same is true for unstable manifolds. However, stable manifolds can intersect unstable manifolds when the flow is unsteady. In this range of $R e$, where the flow is steady, there are three distinct regions 
of the phase portrait. These regions are separated by the stable and unstable manifolds, which coincide for steady flows, between which there is no transport. The two regions enclosing the elliptic points $C_{2}$ and $C_{3}$ (see figure $3 a$ ) are commonly referred to as vortex breakdown bubbles.

As shown in figure 2, at a Reynolds number in the range 2300-2400, the two bubbles coalesce and there is a bifurcation in the topology of the flow. The two hyperbolic fixed points $P_{2}$ and $P_{3}$ (figure $3 a$ ) on the axis are replaced by a single hyperbolic fixed point $S_{1}$ off the axis, which has a double homoclinic orbit associated with it enclosing the two elliptic fixed points $C_{2}$ and $C_{3}$ (figure $3 b$ ). The fixed points $P_{1}, P_{2}, P_{3}$ and $P_{4}$ in figure $3(a)$ are also fixed points of the flow (i.e. stagnation points), however, the fixed point $S_{1}$ in figure $3(b)$ is a periodic point of the flow, its period being determined by the azimuthal velocity at that point. The two separate heteroclinic orbits of figure $3(a)$ are replaced by a single heteroclinic orbit between $P_{1}$ and $P_{4}$. It is clear from figure 2 that at $R e=2500$ the homoclinic and heteroclinic orbits are extremely close.

\subsection{Flow transients}

Figure 4 shows the time history of the stream function at the point $r=\frac{1}{6} R, z=\frac{2}{3} H$ for $H / R=2.5$ and for a series of $R e$ covering the bifurcation depicted in figure 3 . When the steady-state flow has two distinct bubbles placed well apart, the transient oscillation resulting from the impulsive start is damped out after 500-1000 endwall rotations (the non-dimensional unit of time used is one rotation of the bottom endwall). For flows closer to the bifurcation point (i.e. $2300<R e<2400$ ) the transient oscillation still damps out in approximately the same time. Lopez (1989) examined the power spectra of these time series and found a new mode of oscillation appearing after approximately 500 endwall rotations. The transient oscillation with frequency $f_{0} \approx 0.0175$, was found to be independent of Reynolds number. The new frequency $f_{1} \approx 0.0278$, is approximately independent of $R e$ over the $R e$ range in which it is present. The instantaneous streamline pattern oscillates with frequency $f_{1}$ between the topology of figure $3(a)$ and figure $3(b)$ before settling on one of the two.

For $R e<2600$ this $f_{1}$ oscillation is also damped and the flows settle down to steady state. Note that in figure 4 the time series for $R e=2600$ has not settled down to a steady state although the oscillations are damped. The calculations in figure 4 correspond to a grid size of 61 radial nodes $\left(n_{r}\right)$ and 151 axial nodes $\left(n_{z}\right)$ with a time step $(\delta t)$ of 0.05 . The $R e=2600$ case (and all the cases with $R e>2600$ ) has been recalculated for this study with $n_{r}=91, n_{z}=226$ and $\delta t=0.04$ and it has been integrated out to $t=6000$, at which time the $f_{1}$ oscillation, whilst still being damped, has reached neither a steady state nor a periodic flow.

\section{The unsteady flow}

\subsection{The Eulerian picture}

Using the numerical technique described in Lopez (1990), solutions for the enclosed swirling cylinder flow have been sought outside the steady regime. For $H / R=2.5$ the following observations were made:

(i) At $R e=2650$ the impulsively started flow is oscillatory with frequency $f_{1}$ and very weakly damped. The long-term solution may or may not be purely periodic. When the time average of one period of the oscillatory solution was taken as the initial condition the flow was found to approach a steady solution.

(ii) For $2650<R e \leqslant 2675$ impulsively started flows approach a periodic solution with frequency $f_{1}$. 

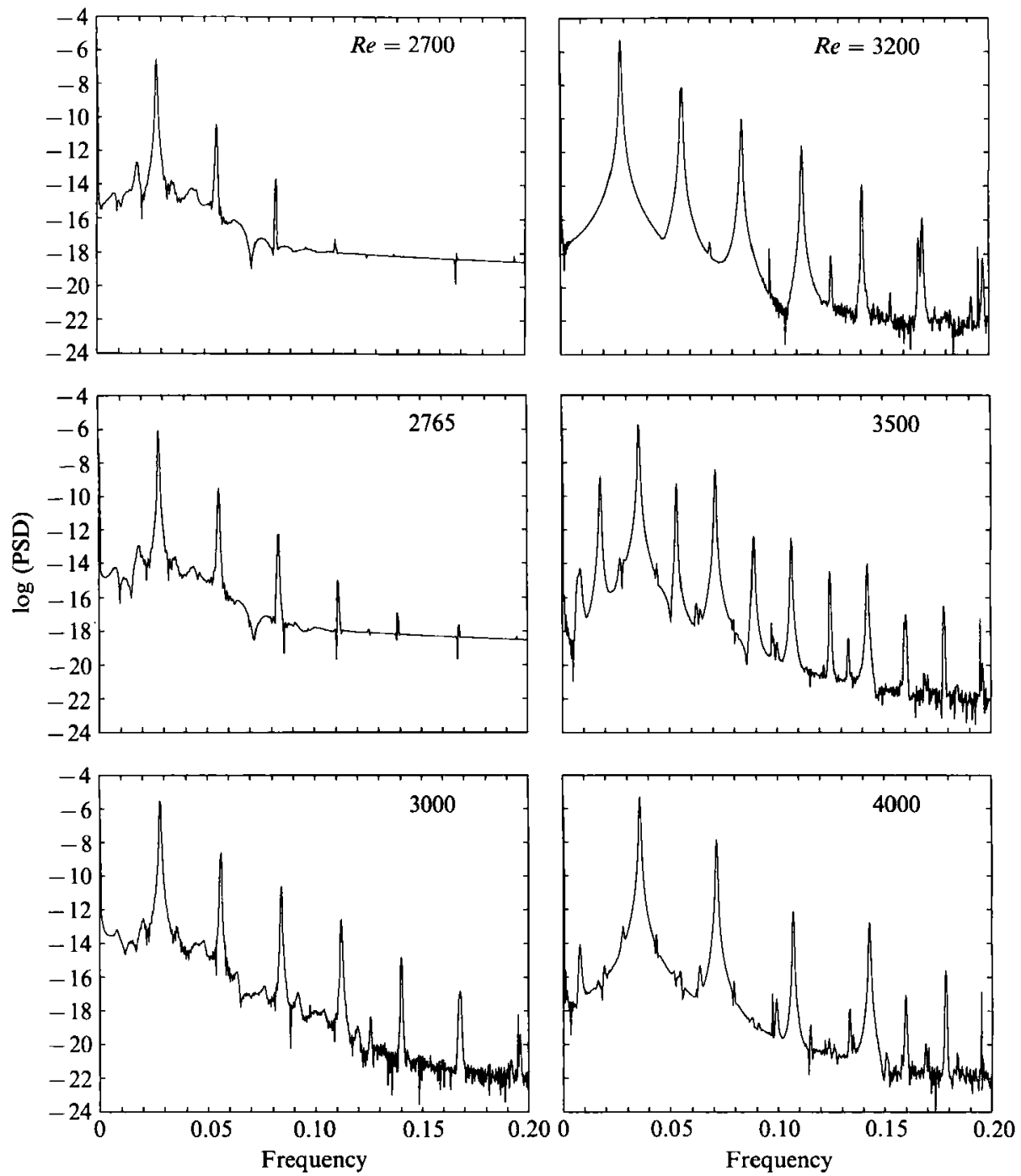

Figure 5. Power spectra of the axial velocity at $r=0, z=\frac{1}{2} H$ for $H / R=2.5$ and $R e$ as indicated. The spectra have been taken over the time interval $6300 \leqslant t \leqslant 8922$ using $2^{16}$ samples $\left(n_{r}=91\right.$, $n_{z}=226$ and $\left.\delta t=0.04\right)$.

(iii) At $R e=2675$ a stable steady solution close to the time average of the periodic solution also exists. This solution was found by taking as the initial condition the time average of the periodic flow.

(iv) For $3500 \leqslant R e<3600$ the long-term solution to impulsively started flows contains $f_{1}$ and $f_{2} \approx 0.0357$ oscillations. The frequencies do not appear to be commensurate.

(v) For $3600 \leqslant R e \leqslant 4000$ impulsively started flows approach purely periodic solutions with frequency $f_{2}$.

Power spectra of the axial velocity at the centre of the cylinder for $2700 \leqslant R e \leqslant$ 4000 are shown in figure 5 .

The Eulerian picture of the $f_{1}$ periodic flows, such as the $R e=2675$ case, consist of two 'bubbles' (closed regions defined by the separatrices of the instantaneous 

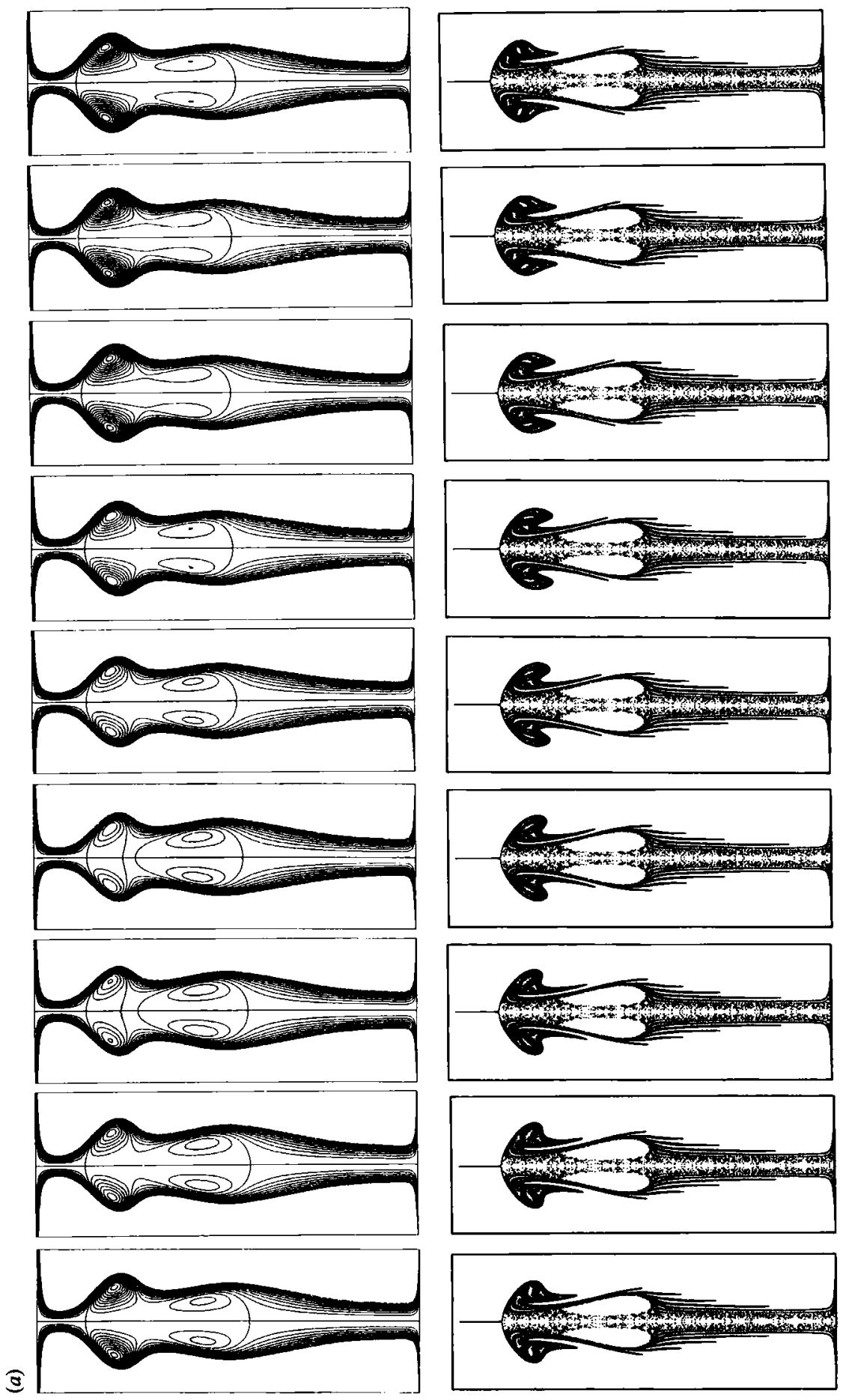

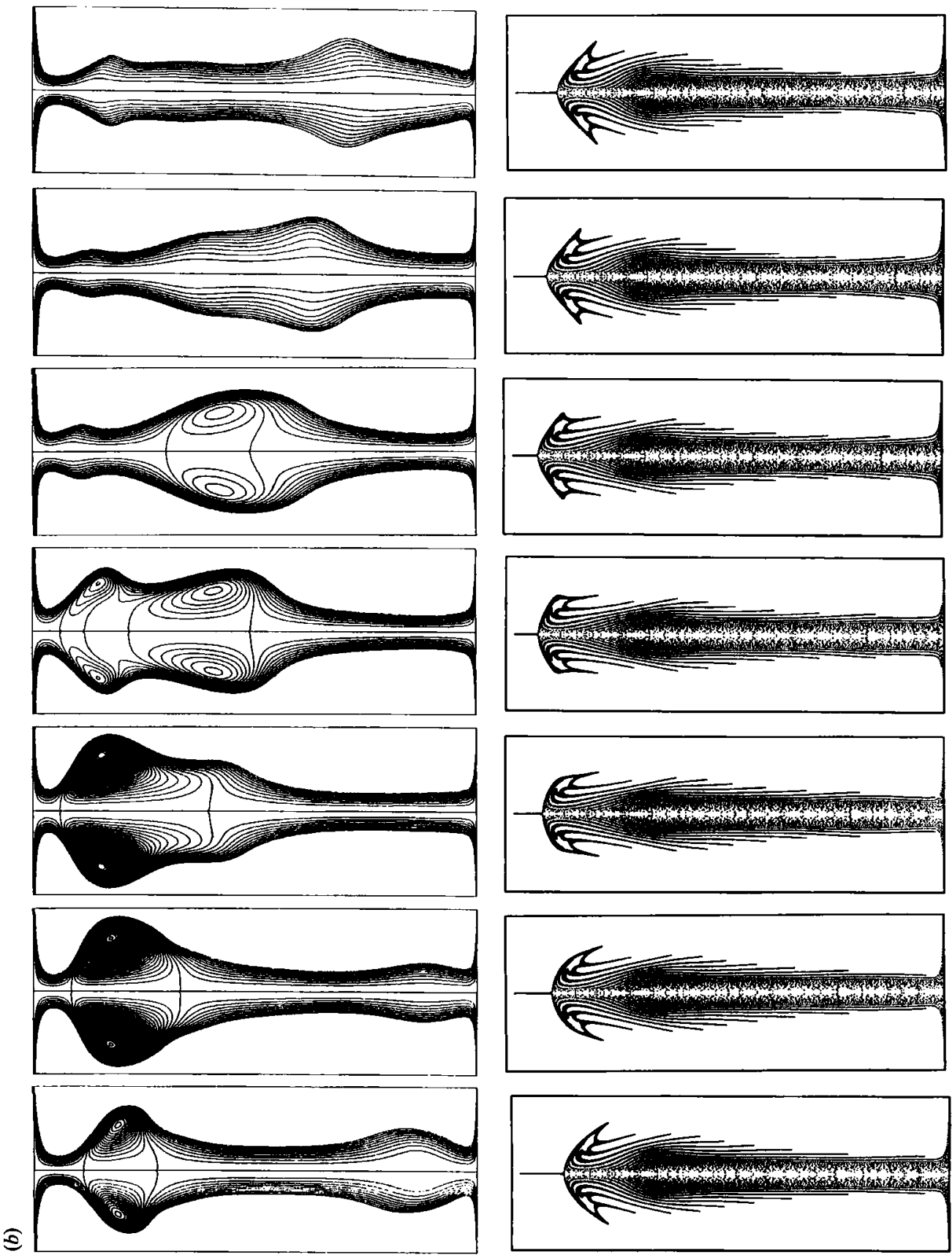

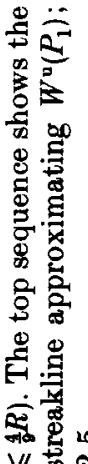

+ 011

$\mathrm{V} / \mathrm{f}$ के

온

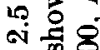

굥ㅇㅇ

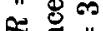

约要

चิ क्षे

120

穴 ‘

II

욜

$8 \stackrel{5}{\circ}$

궁

5

蔚

尊

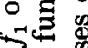

踇

需合

岁密

of on

楼

동

实

혈

范

을

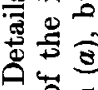

호의

10

0.5

월

田

홍 
stream function) coalescing and separating with frequency $f_{1}$, as in the transients of the steady flows described above. This is shown in the instantaneous streamline patterns of figure $6(a)$. For the $f_{2}$ periodic flows occurring at higher Reynolds numbers, such as $R e=3600$ (figure $6 \mathrm{~b}$ ), the Eulerian picture consists of a 'bubble' moving from the top of the cylinder to the bottom once each period. Relative to a stationary observer, the closed instantaneous streamlines of the 'bubble' disappear during its motion down the cylinder and are replaced by a travelling wave.

\subsection{Transverse intersections of the stable and unstable manifolds}

When the dynamical system (2) is time-dependent and its associated Poincaré map has hyperbolic fixed points, the stable and unstable manifolds of these fixed points need no longer coincide but may intersect each other transversely (i.e. nontangentially). The point of intersection belongs to two distinct invariant curves, the stable and unstable manifolds, and as such, will always belong to them. Intersection points map to intersection points but not to themselves (they are not fixed points). Hence, one such intersection implies infinitely many; the intersection points must therefore accumulate near the fixed points. The points of intersection are transverse homoclinic or heteroclinic points, depending on whether the stable and unstable manifolds correspond to the same (homoclinic) or different (heteroclinic) hyperbolic fixed points.

Without an analytical description of the flow it is difficult to find the stable and unstable manifolds in the unsteady regime. However, good approximations of the unstable manifolds can be obtained both numerically and from flow visualization experiments. For example, at $R e=2765$ there is an unstable manifold emerging from a hyperbolic fixed point on the axis which is delineated by a streakline released close to the axis and near the top endwall. This is shown experimentally (Escudier 1984) in figure $7(b)$ and numerically in figure $7(c)$. Figure $7(a)$ shows the unstable manifold for $R e=2700$. The experiment, with $R e$ estimated at 2765 , seems to correspond to a numerical solution with $R e$ between 2700 and 2765. This small discrepancy in $R e$ between experiment and computation can be partially explained by the $0.5 \%$ uncertainty in the experimental estimate of the viscosity (Lopez 1990) and partially by the error in numerically resolving the rotating-endwall boundary layer with thickness of order $R e^{-\frac{1}{2}}$. It must also be remembered that the streakline patterns are phase-dependent and it is difficult to match the phase of the experiment.

Stable manifolds cannot be visualized experimentally, but remain important for a complete analysis of the flow kinematics. Fortunately, the stable manifolds can be computed numerically. This is achieved by constructing a 'time-reversed' flow for one period of the computed flow by reversing the direction of the velocity field at each time step and by reversing the order of the time steps. In essence, to compute the stable manifold of a hyperbolic fixed point on the axis, particles are released in the 'time-reversed' flow in the neighbourhood of the fixed point.

The unstable manifolds of a hyperbolic fixed point off the axis are found by tracking a 'blob' of particles released simultaneously in its vicinity. The 'blob' is stretched along the unstable manifolds. The stable manifolds are found by using the 'time-reversed' flow.

\subsection{Resonant bands, KAM tori and cantori}

In a steady flow, an elliptic fixed point is surrounded by a sequence of closed streamlines or invariant closed curves. Orbits on these invariant closed curves have an associated period $T_{\mathrm{i}}$, the time a particle on a particular curve takes to complete 
(a)

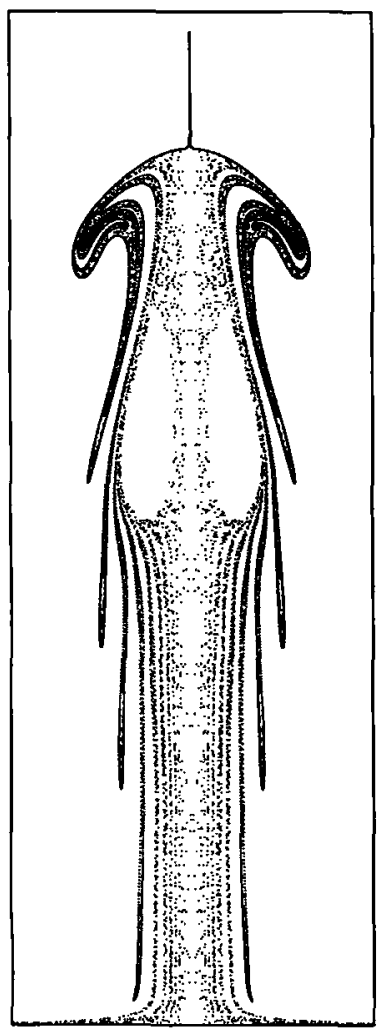

(b)

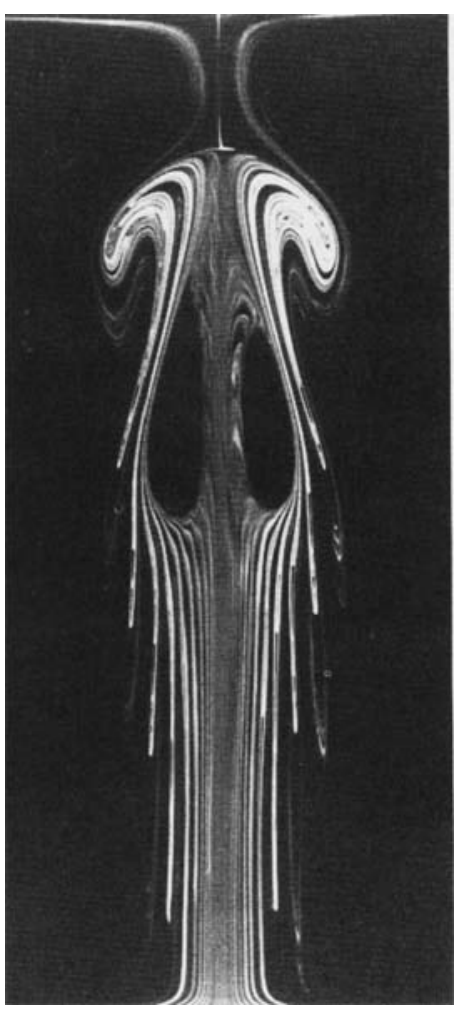

(c)

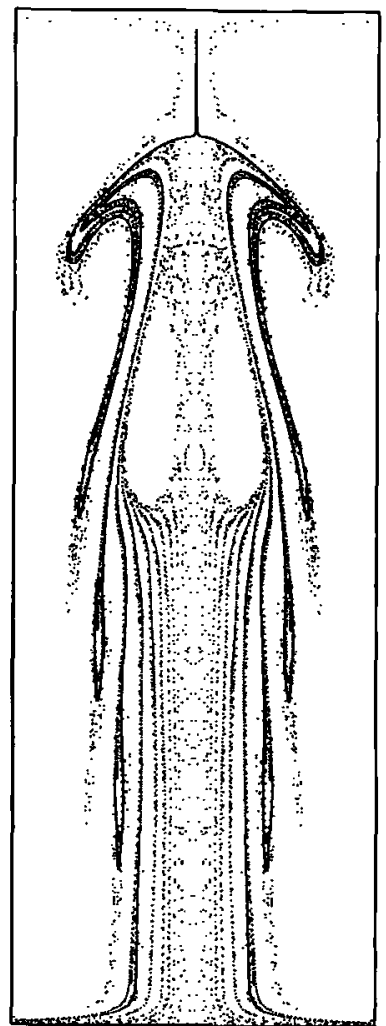

FigURE 7. Unsteady streaklines approximating $W^{\mathrm{u}}\left(P_{1}\right)$ for $(a) R e=2700$ and $(c) R e=2765$, and $(b)$ a snapshot of an unsteady dyeline introduced at $r=0, z=H$ for Re estimated at 2765 (photograph of the experimental visualization is reproduced by kind permission from M. P. Escudier).

one circuit. The behaviour of these orbits in the presence of a small amount of unsteadiness depends on whether the ratio of the period of the orbit to the period of the oscillation $T_{i} / \tau$, the rotation number, is rational or irrational.

If the rotation number is rational then the Poincaré-Birkhoff theorem (see Ottino 1989 or Guckenheimer \& Holmes 1986) states that these rational invariant curves, or resonant tori, will tend to break up into a collection of elliptic and hyperbolic periodic points. These $n$ th-order periodic points are fixed points of $F^{n}$, i.e. they map to themselves every $n$ applications of the Poincaré map. The hyperbolic points have associated stable and unstable manifolds which may intersect transversely leading to the formation of resonant bands or stochastic layers or heteroclinic tangles as the flow becomes unsteady. These thin layers of chaotic motion may be surrounded by invariant curves with irrational rotation numbers. For these curves, the KolmogorovArnol'd-Moser theorem (the KAM theorem) states that if the rotation number is 'sufficiently irrational' then 'most' of the closed invariant curves of the steady flow are preserved as the flow becomes unsteady (see Guckenheimer \& Holmes 1986 for details). These preserved closed invariant curves are referred to as $K A M$ tori and are barriers across which fluid cannot flow. So even in an unsteady flow with mixing due to transverse homoclinic and heteroclinic orbits, there may still be regions of trapped fluid bordered by KAM tori. Such regions of truly trapped fluid may bear little resemblance to the instantaneous streamline 'bubbles'. If the rotation number is neither rational nor 'sufficiently irrational' then as the flow becomes unsteady, an 
invariant closed curve may break down into an invariant cantor set or cantorus (Percival 1979; see Guckenheimer \& Holmes 1986, p. 304 for the conditions leading to the formation of a cantorus). Cantori provide only partial barriers to the flow. In most cases, flow between regions separated by cantori is very slow and it may take many periods before any distinction between KAM tori and cantori can be made.

Although the existence of KAM tori, cantori and resonant bands has only been proven for small perturbations to the steady flow, numerical experiments, including the current investigation, suggest their existence in substantially unsteady flows.

\subsection{Chaotic advection in the central vortical core region}

Fixed points such as those in the core region of the steady flow (figure $3 b$ ) were also found in the $f_{1}$ periodic flow regime, namely a hyperbolic fixed point $S_{1}$, two elliptic fixed points $C_{2}$ and $C_{3}$, and two hyperbolic fixed points on the axis of symmetry $P_{1}$ and $P_{4}$. In the $f_{2}$ periodic regime the core region consists of just two hyperbolic fixed points on the axis and a single elliptic fixed point. As examples of the $f_{1}$ and $f_{2}$ periodic flows, computed approximations of $W^{\mathrm{u}}\left(P_{1}\right)$ and $W^{\mathrm{s}}\left(P_{4}\right)$ for $R e=2675$ and 3600 are shown in figures $8(a), 8(b), 9(a)$ and $9(b)$. Distorted but topologically correct diagrams of all the stable and unstable manifolds in the core region are shown in figures $8(c)$ and $\mathbf{9}(c)$. For clarity the two elliptic fixed points, $C_{2}$ and $C_{3}$, are omitted in figure $8(c)$.

We make the following observations for the $f_{2}$ periodic case, $R e=3600$ :

(i) $W^{\mathrm{u}}\left(P_{1}\right)$ and $W^{\mathrm{s}}\left(P_{4}\right)$ intersect transversely (figure 9 ) and are folded and stretched to form long thin fingers. The fingers of the unstable manifold are stretched towards the rotating endwall. The fingers of the stable manifold draw fluid from upstream into the 'bubble' (as defined below).

(ii) There is a region around the elliptic fixed point $C_{4}$ which the manifolds envelope but do not penetrate (figure 9 ). It appears that this region must be bordered by a KAM torus or cantorus.

The core flow can be divided into regions by the choice of a convenient primary intersection point (Rom-Kedar \& Wiggins 1990), such as the point $p$ (figure $9 c$ ). Let the region enclosed by $P_{1} p P_{4} P_{1}$ (drawn with a thick line), be $R_{2}$ and let its complement be $R_{1}$. The vortex breakdown bubble, defined as region $R_{2}$, oscillates up and down the axis a small distance of the order of half its radius. This is in contrast to the instantaneous streamline 'bubble' which travels the full length of the axis (see figure $6 b$ ).

Intersection points of $W^{\mathrm{u}}\left(P_{1}\right)$ and $W^{\mathrm{s}}\left(P_{4}\right)$ are mapped to other intersection points. For example, $a$ is mapped to $p$ and $b$ is mapped to $q$. This means that the shaded region or lobe (Rom-Kedar \& Wiggins 1990) $L_{2,1}$, is mapped to $F\left(L_{2,1}\right)$. The subscripts on $L_{2,1}$ indicate that it is the lobe to be mapped from $R_{2}$ to $R_{1}$ upon the next application of the Poincaré map. To preserve area (in $(p, z)$-space, the lobe entering the bubble must have the same area as the one leaving, i.e. $L_{1,2}$ and $L_{2,1}$ have the same area. All images of a lobe under iterations of the map are of equal area, e.g. $L_{2,1}$, $F\left(L_{2,1}\right), F^{2}\left(L_{2,1}\right), \ldots$ are all of the same area. The accumulation of intersection points at the hyperbolic fixed points means that the lobes stretch to maintain their area, thus forming long thin fingers. This phenomenon is absent in steady flows since the stable and unstable manifolds do not intersect transversely.

Information such as residence times and the extent of mixing for a given period can be deduced from the lobe intersections (Rom-Kedar \& Wiggins 1990). For example, it can be deduced that the shaded region $X$, in figure $9(c)$, was outside the bubble four periods ago and will be outside again in another three. 

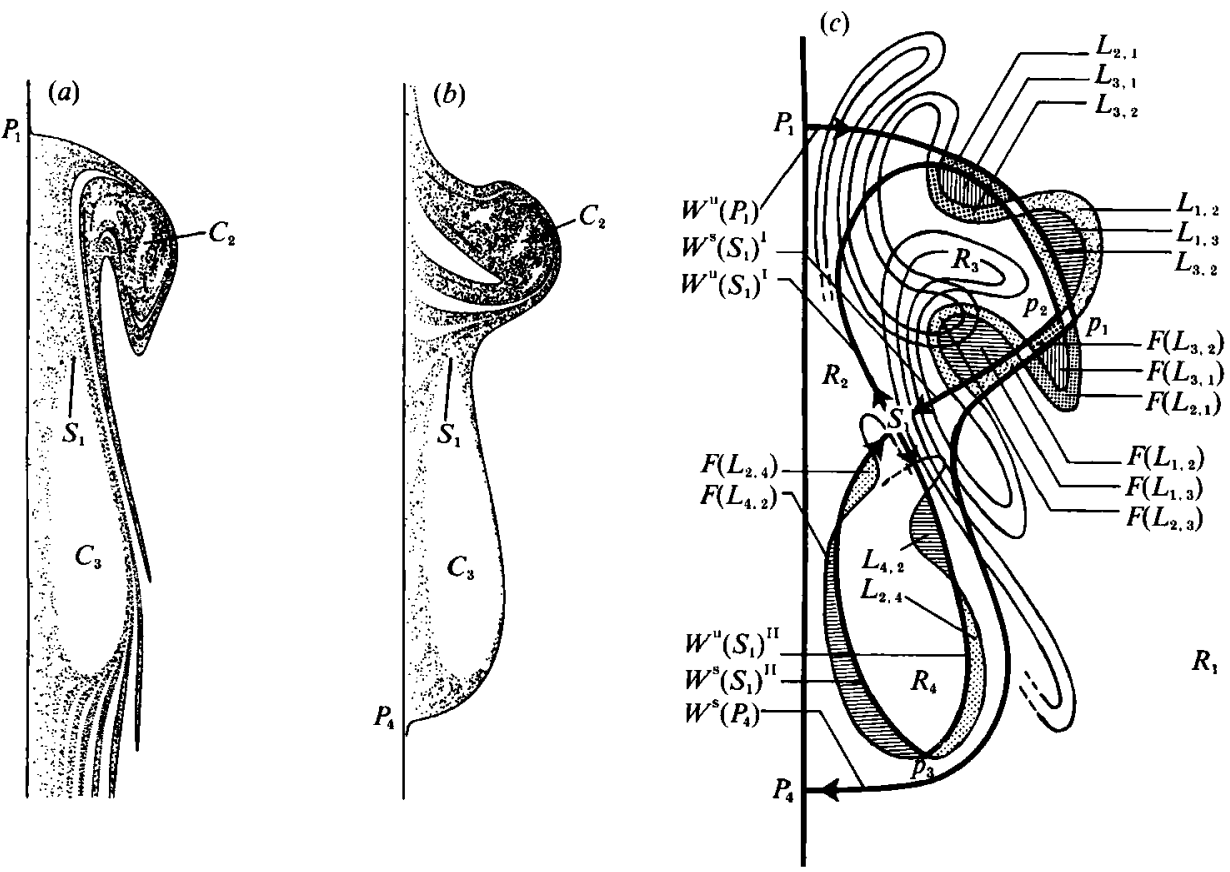

FIGURE 8. (a) A streakline upstream of $P_{1}$, giving an approximation of $W^{\mathrm{u}}\left(P_{1}\right)$ at $R e=2675$ and $H / R=2.5$ and $(b)$ a streakline released in the 'time-reversed' flow near $P_{4}$, giving an approximation of $W^{\mathrm{s}}\left(P_{4}\right)$. (c) A schematic of $W^{\mathrm{u}}\left(P_{1}\right), W^{\mathrm{u}}\left(S_{1}\right)^{\mathrm{I}}, W^{\mathrm{u}}\left(S_{1}\right)^{\mathrm{n}}, W^{\mathrm{s}}\left(P_{4}\right), W^{\mathrm{s}}\left(S_{1}\right)^{\mathrm{I}}$ and $W^{\mathrm{s}}\left(S_{1}\right)^{\mathrm{II}}$ for a typical $f_{1}$ oscillation.
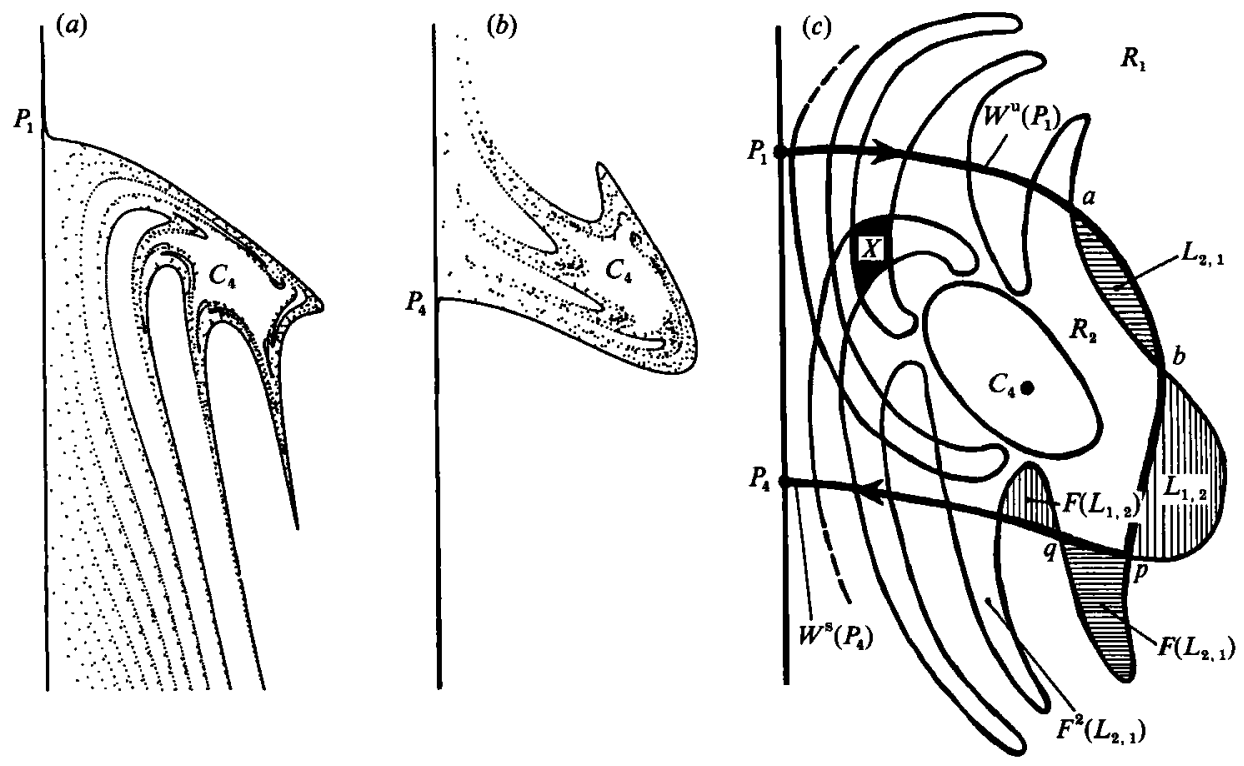

Figure 9. (a) A streakline released upstream of $P_{1}$, giving an approximation of $W^{\mathrm{u}}\left(P_{1}\right)$ for $R e=$ 3600 and $H / R=2.5$ and $(b)$ a streakline released in the 'time-reversed' flow near $P_{4}$, giving an approximation of $W^{s}\left(P_{4}\right)$. (c) A schematic of $W^{\mathrm{u}}\left(P_{1}\right)$ and $W^{s}\left(P_{4}\right)$ for a typical $f_{2}$ oscillation. 
Heteroclinic tangles with similar topologies have been studied in detail by RomKedar et al. (1990).

The kinematics of the $f_{1}$ periodic flows is more complicated. We make the following observations for $R e=2675$ :

(i) One unstable manifold emerging from $S_{1}\left(W^{\mathrm{u}}\left(S_{1}\right)^{\mathrm{I}}\right.$ in figure 8) coils up around the elliptic fixed point $C_{2}$, to subsequently run alongside $W^{\mathrm{u}}\left(P_{1}\right)$ (which it cannot intersect).

(ii) The two unstable manifolds $W^{\mathrm{u}}\left(S_{1}\right)^{\mathrm{I}}$ and $W^{\mathrm{u}}\left(P_{1}\right)$, intersect transversely with both $W^{\mathrm{s}}\left(S_{1}\right)^{\mathrm{I}}$ and $W^{\mathrm{s}}\left(P_{4}\right)$. This results in folding and stretching of the two unstable manifolds at $S_{1}$ and $P_{4}$, forming long fingers. These fingers are stretched both towards the rotating endwall and towards $P_{1}$, where they follow $W^{\mathrm{u}}\left(P_{1}\right)$ and fit inside the new lobes forming from $W^{\mathrm{u}}\left(P_{1}\right)$ (figure 8).

(iii) There are at least three regions in the vicinity of $C_{2}$ which the folds of the manifolds envelope but do not penetrate (figure 8). The central one 'rotates' on the spot while the two satellite regions swap each period. The central region containing $C_{2}$ is apparently bordered by a KAM torus or cantorus (which is invariant under the Poincare map). The satellites imply the existence of two elliptic periodic points of period two, i.e. these points map to themselves under two applications of the Poincaré map. Each is surrounded by a KAM torus (or cantorus) invariant under a double application of the Poincaré map. At higher $R e$, e.g. $R e=2700$ and 2765 (see figure 8), these islands occupy smaller regions of phase space.

(iv) It appears that $W^{\mathrm{u}}\left(S_{1}\right)^{\mathrm{II}}$ and $W^{\mathrm{s}}\left(S_{1}\right)^{\mathrm{II}}$ intersect transversely, but only just; the lobes formed are of very small area. There is a large KAM torus or cantorus surrounding the elliptic fixed point $C_{3}$ (in figure 8). The region separating $S_{1}$ and the torus is very thin.

The $C_{2}$ elliptic region shrinks as $R e$ is increased. The elliptic fixed point becomes increasingly difficult to locate. Can it disappear altogether? The topological rule formulated in the Appendix implies that the elliptic fixed points must remain unless there is a corresponding change in the number of hyperbolic fixed points. This was not observed and it is concluded that the elliptic fixed point remains.

The core flow has been divided into four regions $R_{1}-R_{4}$ using the primary intersection points $p_{1}, p_{2}$ and $p_{3}$ (figure $8 c$ ). A lobe about to move from one region to another and its location in the new region are shaded in the same pattern. For example, $L_{2,1}$ moves from inside $R_{2}$ to inside $R_{1}$. Regions $R_{1}, R_{2}$ and $R_{3}$ each exchange fluid with each other every period. In one period $R_{4}$ only exchanges fluid with $R_{2}$. Most of $R_{4}$ is made up of an unmixed (or slowly mixing) sub-region bordered a KAM torus (or cantorus). This is in stark contrast to $R_{3}$, which undergoes rapid mixing.

\subsection{The meridional circulation}

Thus far the discussion of the flow inside a cylinder driven by a rotating endwall has been limited to the central vortex core region which undergoes vortex breakdown. It is also of interest to examine the meridional circulation.

In the steady flows, as shown schematically in figure 3 , the meridional circulation results in a Poincaré map consisting of an elliptic fixed point $C_{1}$ and a series of closed invariant orbits about $C_{1}$. From the Poincaré-Birkhoff theorem, some of these orbits (the rational orbits) are likely to break up into a series of elliptic and hyperbolic periodic points as the Reynolds number is increased and the flow becomes unsteady.

At $R e=2765$, one such resonant band has been found, consisting of four elliptic and four hyperbolic periodic points of order four, $E_{4}^{1}-E_{4}^{4}$ and $H_{4}^{1}-H_{4}^{4}$. Each period, $E_{4}^{1}$ is mapped to $E_{4}^{2}, E_{4}^{2}$ is mapped to $E_{4}^{3}, E_{4}^{3}$ is mapped to $E_{4}^{4}$ and $E_{4}^{4}$ is mapped to $E_{4}^{1}$. 


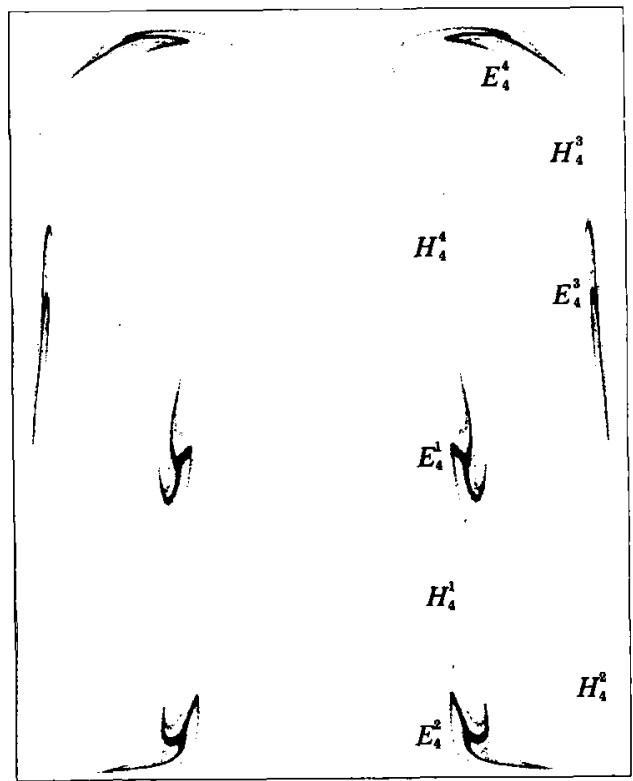

(a) 55 applications of the map

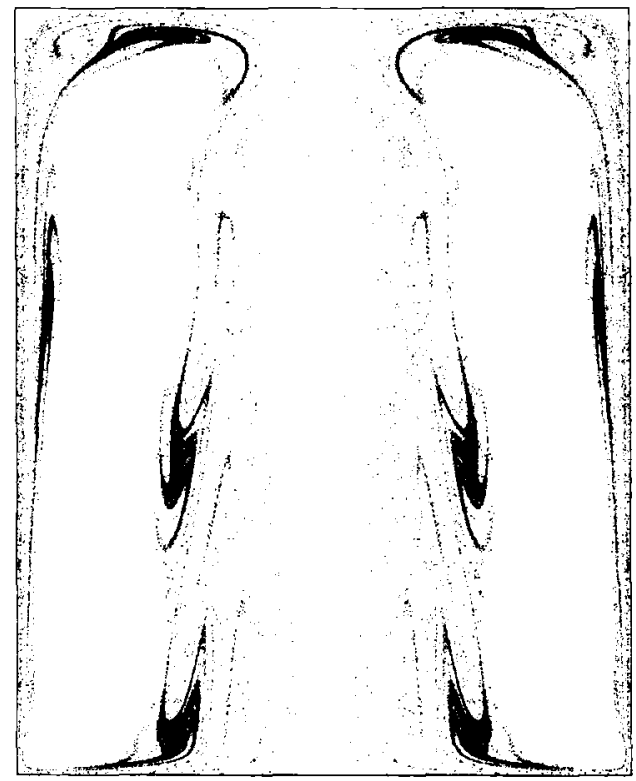

(c) 91 applications of the map

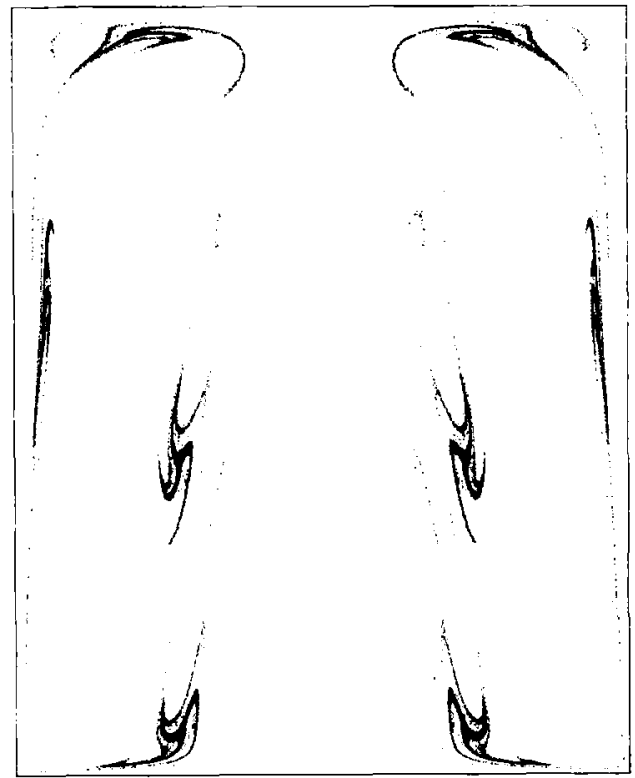

(b) 67 applications of the map

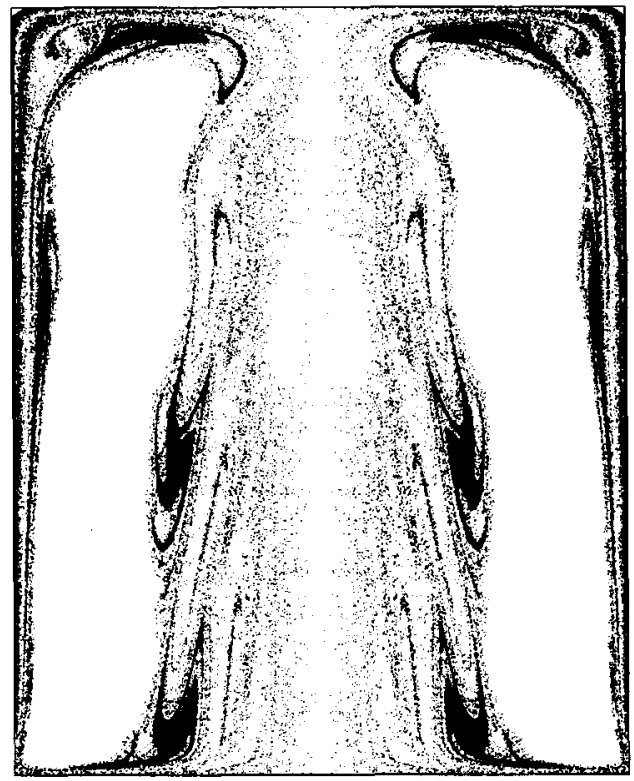

(d) 152 applications of the map

Figure 10. Phase portraits revealing the resonance band in the meridional circulation for $R e=$ 2765 and $H / R=2.5$. A 'blob' is released in the neighbourhood of $E_{4}^{1}$ and a montage is built up using its images from a number of applications of the Poincaré map.

The hyperbolic points behave similarly (see figure 10a). The stable and unstable manifolds of these hyperbolic periodic points intersect transversely, leading to the formation of a resonance band, as depicted in figure 10 .

A numerical estimate of the Poincare map is obtained by releasing a large number of particles uniformly over the whole of phase space and noting where they are 


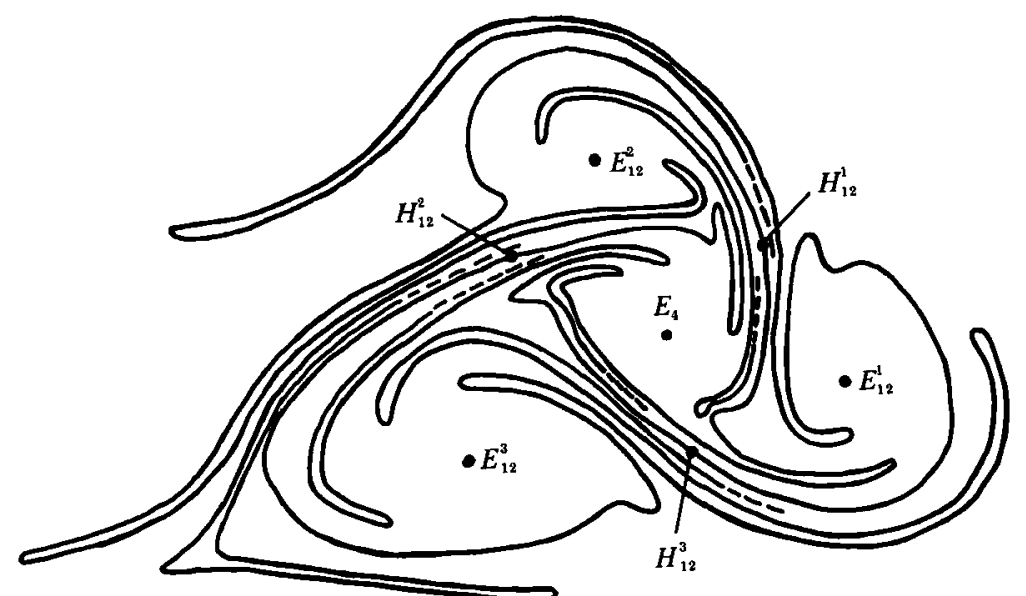

Fraure 11. A schematic of the period-twelve resonance band about a period-four elliptic point found in the meridional circulation for $R e=2765, H / R=2.5$.

advected to after one period. This was done for the case $R e=2765$ with $n_{r}=91$, $n_{z}=226$ and $\delta t=0.04$. A total of $2028151(901 \times 2251)$ particles were tracked for one period $(900 \delta t)$, giving a reasonably smooth estimate of the Poincaré map for this case. From this map, a scalar function can be defined which corresponds to the distance a particle is displaced after one iteration of the Poincaré map. The zeros of this function give the location of all the fixed points. Also, if this map is applied $n$ times to all the original points, then the zeros of the distance function give not only the location of the fixed points, but also the location of the periodic points of order $n / m$, where $m$ and $n / m$ are integers.

The phase portraits depicting the resonance band in figure 10 were obtained by locating the periodic points of order four using the above technique and releasing a dense 'blob' of 3600 particles in the neighbourhood of one of these elliptic periodic points. The portraits in figure 10 are montages of the image of this 'blob' after successive applications of the map.

Closer examination of the elliptic regions surrounding $E_{4}^{1}-E_{4}^{4}$, reveals higher-order periodic points. About each of $E_{4}^{1}-E_{4}^{4}$, there exists a resonance band consisting of three elliptic and three hyperbolic periodic points of order twelve, $E_{12}^{1}-E_{12}^{3}$ and $H_{12}^{1}-H_{12}^{3}$. These are shown schematically in figure 11. Also shown in figure 11 are the unstable manifolds from $H_{12}^{1}-H_{12}^{3}$. On each four-fold application of the map, $E_{12}^{1}$ is mapped to $E_{12}^{2}, E_{12}^{2}$ is mapped to $E_{12}^{3}$ and $E_{12}^{3}$ is mapped to $E_{12}^{1}$ and similarly for the points $H_{12}^{1}-H_{12}^{3}$.

Recall from figure $7(c)$ that there is an unstable manifold emanating from the hyperbolic fixed point on the axis, $W^{\mathrm{u}}\left(P_{1}\right)$. It takes about 100 periods for the fluid in the central core region to mix with the meridional circulation. The only way this mixing can occur is for the lobes formed by $W^{\mathrm{u}}\left(P_{1}\right)$ to weave their way amongst the folds created by the heteroclinic tangles of the unstable manifolds from $H_{4}^{1}-H_{4}^{4}$. This is indicated in figure $10(d)$.

\section{Conclusions}

A typical sequence of bifurcations has been studied for the flow inside a cylinder, driven by a rotating endwall, with aspect ratio 2.5 . 
The steady regime consists of three topologies. At low $R e$, the flow consists of a simple swirling motion with a meridional circulation (one elliptic fixed point). At higher $R e$ there are two separate bubbles on the axis (one elliptic fixed point for the meridional circulation and two hyperbolic fixed points and an elliptic fixed point for each bubble). Just prior to the onset of periodic flow, the bubbles coalesce (one elliptic fixed point for the meridional circulation, a hyperbolic fixed point with a double homoclinic connection enclosing two elliptic fixed points and two hyperbolic fixed points on the axis).

The Eulerian picture of the low-Re end of the unsteady regime consists of two 'bubbles' (closed regions defined by the separatrices of the instantaneous streamline pattern) coalescing and separating at frequency $f_{1}$. The fixed points of the Poincaré map are the same as that of the steady flow just prior to the onset of unsteadiness. The transverse intersections of the stable and unstable manifolds lead to a complicated Lagrangian picture of the central core flow. At higher Reynolds numbers, the Eulerian picture has changed to that of a 'bubble', or wave, travelling from one end of the cylinder to the other with frequency $f_{2}$. This bifurcation is reflected in a change in the Lagrangian picture, where the two elliptic fixed points and the associated hyperbolic fixed point are replaced by a single elliptic fixed point.

One of the most important consequences of this study is a clear understanding of the filling and emptying process of the vortex breakdown 'bubbles', in this particular geometry. The Eulerian picture of this process is inadequate and the process only becomes understandable when viewed in terms of the transverse intersections of the stable and unstable manifolds. Whilst flow visualization provides a very good representation of the unstable manifolds, it is only via numerical calculations that the stable manifolds can be determined in highly nonlinear flows with no known analytical solutions, such as vortex-breakdown swirling flows.

Holmes' (1984) prediction of the fluid motion in the vortex-breakdown bubble consisting of thin fingers originating upstream entering the bubble, where they circulate at least once, and similarly fluid originating inside the bubble exiting via thin fingers, is the type of motion found here. For the particular geometry studied here, this filling and emptying process is found in an unsteady axisymmetric flow, whereas Holmes used an asymmetric periodic perturbation of the steady axisymmetric vortex-breakdown flow to explain the filling and emptying process.

This study has been a good example of the application of Hamiltonian dynamical systems theory in the interpretation of the complicated behaviour observed in a very unsteady vortical flow at moderate Reynolds numbers, albeit in a simple geometry.

\section{Appendix}

In the neighbourhood of a fixed point $x_{0}$, the Poincaré map $x \mapsto F(x)$ can be described by

$$
F(x)-x_{0}=\mathbf{L}\left(x-x_{0}\right)+O\left(\left|x-x_{0}\right|^{2}\right),
$$

where $\mathbf{L}$ is a linear operator (two-dimensional matrix). For an area- and orientationpreserving map $\operatorname{det}(L)=1$. If $-2<\operatorname{trace}(L)<2$, the fixed point $x_{0}$ is Liapunov stable and called elliptic. If trace $(L)= \pm 2$, it is degenerate and parabolic. Otherwise it is unstable and hyperbolic.

A zero point of the velocity field (i.e. where the velocity is zero) can be classified by the type of critical point it generates in the instantaneous streamline pattern (Perry \& Fairlie 1974). Recall that the streamlines are the set of curves tangent to the velocity field. There is a unique streamline passing through each point in the field 


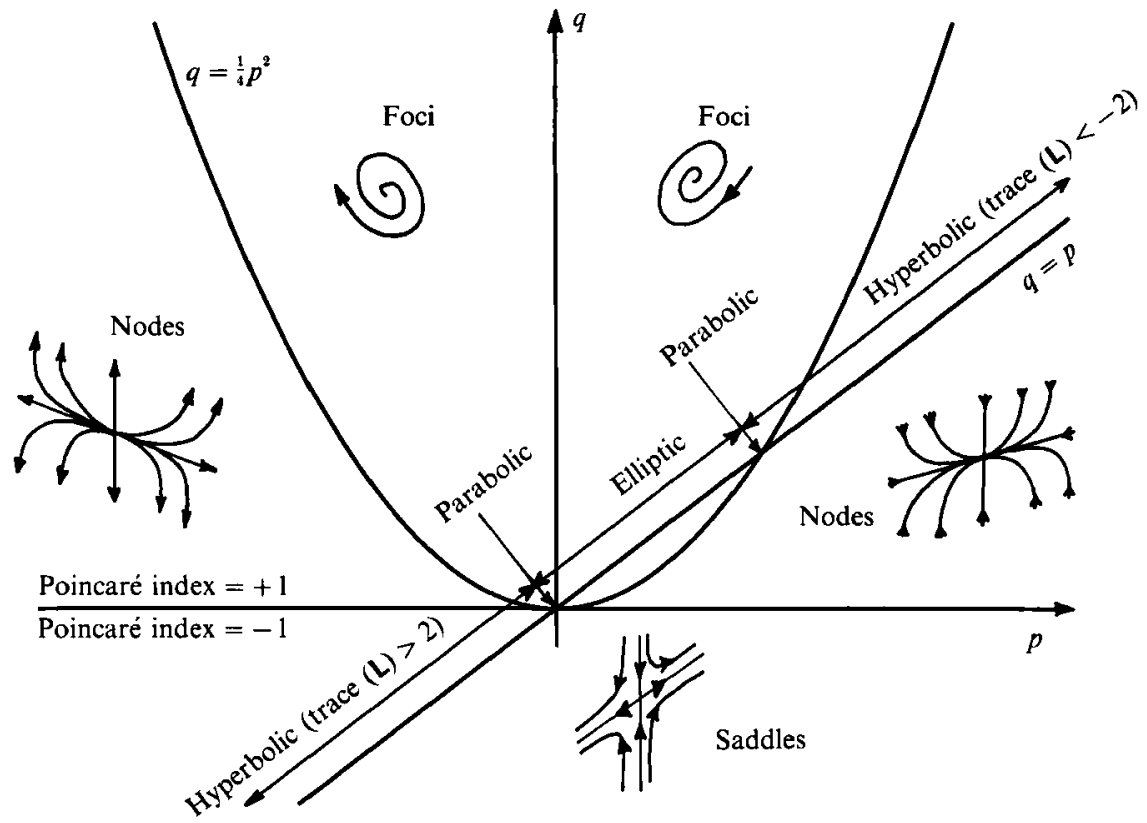

Figure 12. Classification of critical points on the $(p, q)$-chart.

except at the zero points where the direction of the velocity is indeterminate. Rules which relate the number of saddles and centres can be formulated for twodimensional flows using the Poincaré-Bendixson theorem (Davey 1961; Hunt et al. 1978).

The fixed points of the Poincaré map $x \mapsto F(x)$, are the zero points of the vector field $F(x)-x$. There is a system of curves analogous to streamlines tangent to the vector field $F(x)-x$. A fixed point of the map results in a critical point in the system of tangent curves. In the vicinity of a fixed point $\boldsymbol{x}_{\mathbf{0}}$,

$$
F(x)-x=(L-I)\left(x-x_{0}\right)+O\left(\left|x-x_{0}\right|^{2}\right),
$$

where $I$ is the identity operator. The critical point corresponding to $x_{0}$ can be classified by the trace and determinant of $L-I$. Let

$$
p=-\operatorname{trace}(\mathbf{L}-\mathbf{I})=-\operatorname{trace}(\mathbf{L})+2 \text {, }
$$

and

$$
q=\operatorname{det}(\mathbf{L}-\mathbf{I})=\operatorname{det}(\mathbf{L})-\operatorname{trace}(\mathbf{L})+1 \text {. }
$$

The critical points can be classified using a $(p, q)$-chart (figure 12) following the scheme of Perry \& Fairlie (1974). The important conclusions are:

(i) Critical points for area- and orientation-preserving maps must lie on the line $q=p$

(ii) All elliptic fixed points correspond to foci.

(iii) Hyperbolic fixed points with trace (L) $>2$ correspond to saddles.

(iv) Hyperbolic fixed points with trace (L) $<-2$ corresponds to nodes.

Note that the arrows in figure 12 indicate the direction of the vector field $F(x)-x$ and not necessarily the stability of the fixed point. Hyperbolic fixed points with trace (L) $<-2$ are a case in point.

In canonical coordinates (i.e. those which transform $\mathbf{L}$ to Jordan form) a hyperbolic fixed point corresponds to stretching in one eigen-direction and compression in the other. In the case trace $(L)<-2$ (corresponding to nodes) there 


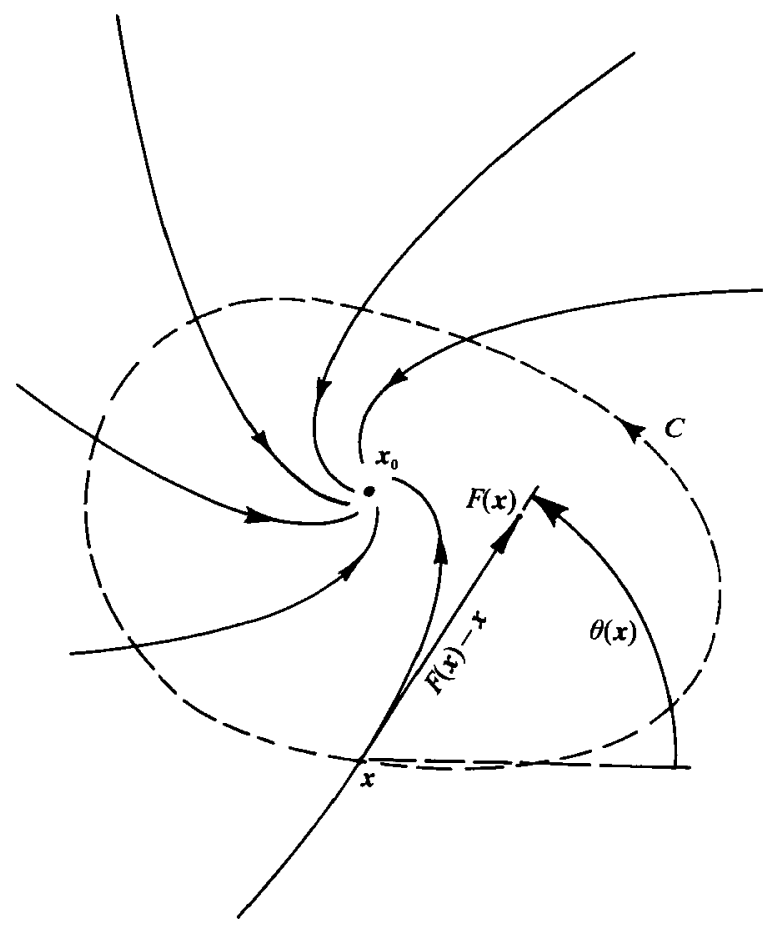

Figure 13. The curves tangent to the vector field $F(x)-x$, in the vicinity of an elliptic fixed point (node).

is a rotation of $\pi$ in addition to the stretching and compression. A particle released on one stable (unstable) manifold branch oscillates between both branches as it approaches (leaves) the fixed point. Hyperbolic fixed points of this kind cannot occur on the axis of an axisymmetric flow since the fixed point is missing one branch. Therefore, all fixed points on the axis must correspond to saddle points.

A heuristic derivation of a rule relating the number of elliptic and hyperbolic fixed points is possible using a Poincaré index approach (Davey 1961; Hunt et al. 1978). Figure 13 shows the system of curves tangent to $F(x)-x$ in the vicinity of an elliptic fixed point $x_{0}$ (node). A circuit $C$ which contains the node is also shown. At each point $\boldsymbol{x}$ on $C$ define $\theta(x)$ to be the angle of deviation of the vector $F(x)-x$ from the horizontal, measured positive in the anticlockwise direction. Let $\Delta \theta$ for $C$ be the change in $\theta$ for an anticlockwise traverse of $C$. The Poincaré index of $C$ is defined as $\Delta \theta / 2 \pi$. It can be seen that the index for any simple closed circuit containing the node will be +1 . On the $(p, q)$-chart the index of a critical point is +1 if $q>0$ and -1 if $q<0$ (figure 12). The index of a circuit containing no critical points is zero. In general the index of a simple closed circuit is the sum of the indices of the critical points contained in the circuit. The Poincaré-Bendixson theory can be extended to nonplanar geometries (Davey 1961).

The Poincare index of a circuit close to the wall and the axis of symmetry of the swirling cylinder flow can be deduced from the behaviour of the vector field $F(x)-x$ near the wall and axis. This can be used to derive a rule for the number of elliptic and hyperbolic fixed points off the wall and axis without knowing any details about the interior flow.

Let it be assumed that no stable or unstable manifolds emerge from the parabolic fixed points on the no-slip boundaries. Consider the circuit $C$, close to the walls and 


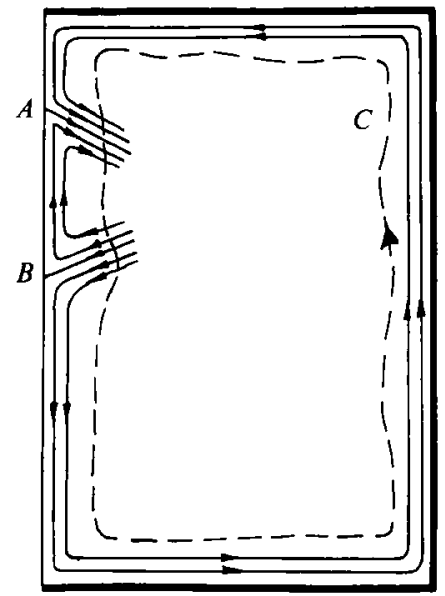

Frgure 14. Schematic of the curves tangent to the vector field $F(x)-x$ close to the walls and axis. Points $A$ and $B$ are saddle points corresponding to hyperbolic fixed points on the axis.

axis, shown in figure 14. Irrespective of what lies inside circuit $C$, its Poincaré index $\mathrm{PI}_{C}$ can be shown to be

$$
\mathrm{PI}_{C}=1+\frac{1}{2} \Sigma_{A}
$$

where $\Sigma_{A}$ is the number of saddles (corresponding to hyperbolic fixed points) on the axis. The sum of the Poincaré indices of the critical points inside $C$ must add to $\mathrm{PI}_{C}$ :

$$
\mathrm{PI}_{C}=\Sigma_{E}-\Sigma_{H^{+}}+\Sigma_{H^{-}}
$$

where $\Sigma_{E}$ is the number of foci (elliptic fixed points), $\Sigma_{H^{+}}$is the number of saddles (hyperbolic fixed points with trace $(L)>2$ ) and $\Sigma_{H^{-}}$is the number of nodes (hyperbolic fixed points with trace $(L)<-2$ ). Equating (A 1) and (A 2) gives the desired rule:

$$
\Sigma_{E}-\Sigma_{H^{+}}+\Sigma_{H^{-}}=1+\frac{1}{2} \Sigma_{A} \text {. }
$$

\section{REFERENCES}

Benjamin, T. B. 1962 Theory of the vortex breakdown phenomenon. J. Fluid Mech. 14, 593-629. Brown, G. L. \& Lopez, J. M. 1990 Axisymmetric vortex breakdown. Part 2. Physical mechanisms. J. Fluid Mech. 221, 553-576.

Daube, O. \& Sorensen, J.N. 1989 Simulation numérique de l'écoulement périodique axisymétrique dans une cavité cylindrique. C. R. Acad. Sci. Paris 308, 463-469.

DAveY, A. 1961 Boundary-layer flow at a saddle point of attachment. J. Fluid Mech. 10, 593-610.

Escudier, M. P. 1984 Observations of the flow produced in a cylindrical container by a rotating endwall. Expts. Fluids 2, 189-196.

Faler, J. H. \& Leibovich, S. 1977 Disrupted states of vortex flow and vortex breakdown. Phys. Fluids 20, 1385-1400.

GUCK ENheImer, J. \& Holmes, P. 1986 Nonlinear Oscillations, Dynamical Systems, and Bifurcations of Vector Fields. Springer.

Hama, F. R. 1962 Streaklines in a perturbed shear flow. Phys. Fluids 5, 644-650.

Holmes, P. 1984 Some remarks on chaotic particle paths in time-periodic, three dimensional swirling flows. Contemp. Maths 28, 393-403.

Hunt, J. C. R., Abell, C. J., Peterka, J. A. \& Woo, H. 1978 Kinematical studies of the flows around free or surface-mounted obstables; applying topology to flow visualization. J. Fluid Mech. 86, 179-200.

Lichtenberg, A. J. \& Lieberman, M. A. 1983 Regular and Stochastic Motion. Springer. 
LOPEZ, J. M. 1988 Vortex breakdown of a confined swirling flow. In Computational Fluid Dynamics. Proc Intl Symp. Comp. Fluid Dyn., Sydney, Australia, August 1987 (ed. G. de Vahl Davis \& C. Fletcher), pp. 493-501. North-Holland.

LOPEZ, J. M. 1989 Axisymmetric vortex breakdown in an enclosed cylinder flow. In 11th Intl Conf. on Numerical Methods in Fluid Dynamics (ed. D. L. Dwoyer, M. Y. Hussaini \& R. G. Voigt). Lecture Notes in Physics, vol. 323, pp. 384-388. Springer.

LoPez, J. M. 1990 Axisymmetric vortex breakdown. Part 1. Confined swirling flow. J. Fluid Mech. $221,533-552$.

LUgt, H. J. \& Aвboud, M. 1987 Axisymmetric vortex breakdown with and without temperature effects in a container with a rotating lid. J. Fluid Mech. 179, 179-200.

NeItzel, G. P. 1988 Streak-line motion during steady and unsteady axisymmetric vortex breakdown. Phys. Fluids 31, 958-960.

Otтino, J. M. 1989 The Kinematics of Mixing: Stretching, Chaos, and Transport. Cambridge University Press.

Perctval, I. C. 1979 Variational principles for invariant tori and cantori. Am. Inst. Phys. Conf. Proc. 57, 302-310.

Perry, A. E. \& Fairlie, B. D. 1974 Critical points in flow patterns. Adv. Geophys. 18B, 229-315.

Rom-Kedar, V., LeONARD, A. \& Wiggins, S. 1990 An analytical study of transport, mixing and chaos in an unsteady vortical flow. J. Fluid Mech. 214, 347-394.

Rom-Kedar, V. \& Wiggins, S. 1990 Transport in two-dimensional maps. Arch. Rat. Mech. Anal. $109,239-298$.

RoNNENBERG, B. 1977 Ein selbstjustierendes 3-KomponentenLaserdoppleraremometer nach dem Vergleichsstrahlverfahren, angewandt für Untersuchungen in einer stationären zylindersymmetrischen Drehströmung mit einen Rückstromgebiet. Max-Planck-Inst. Bericht 20.

Stuart, J. T., Pankhurst, R. C. \& Bryer, D. W. 1963 Particle paths, filament lines and streamlines. NPL Aero Rep. 1057.

VOGEL, H. U. 1968 Experimentelle Ergebnisse über die laminare Strömung in einen zylindrischen Gehäuse mit darin rotierender Scheibe. Max-Planck-Inst. Bericht 6.

Wiggins, S. 1988 Global Bifurcations and Chaos: Analytical Methods. Springer.

Wigarns, S. 1990 Introduction to Applied Nonlinear Dynamical Systems and Chaos. Springer. 